\title{
UTILISING DYNAMIC FACTORY SIMULATION TO IMPROVE UNIT COST ESTIMATION AND AID DESIGN DECISIONS
}

\author{
Stuart Jinks \\ Jim Scanlan \\ Philippa Reed \\ University of Southampton \\ Highfield, Southampton \\ Hampshire, SO17 1BJ, UK
}

Steve Wiseall

Rolls-Royce plc

PO Box 31, Derby

Derbyshire, DE24 8BJ, UK

\begin{abstract}
Utilising dynamic simulation methods to estimate manufacturing resources, can improve unit cost estimation and aid design decisions. This paper introduces a framework specification that combines Computer Aided Design (CAD), Computer Aided Process Planning (CAPP) and Discrete Event Simulation (DES) technologies. The framework is used to aid a design team in understanding the consequences of design decisions in terms of cost and manufacturing resources, by returning unit cost and manufacturing based results, directly to the design team, within the design environment. Dynamic Resource Estimation System (DRES) is a system being developed to implement the framework and is presented in this paper.
\end{abstract}

\section{INTRODUCTION}

A Product Development Process (PDP) represent the life cycle of a product from conception of the initial idea, through design, manufacture, operation and finally disposal. A PDP typically contains a number of stages that can be classified into categories. The Rolls-Royce PDP (Rolls-Royce Plc, 2005), illustrated in Figure 1, contains seven stages and three categories. This paper will focus specifically on stage 1, developing the preliminary design of a product that fulfils a business opportunity.

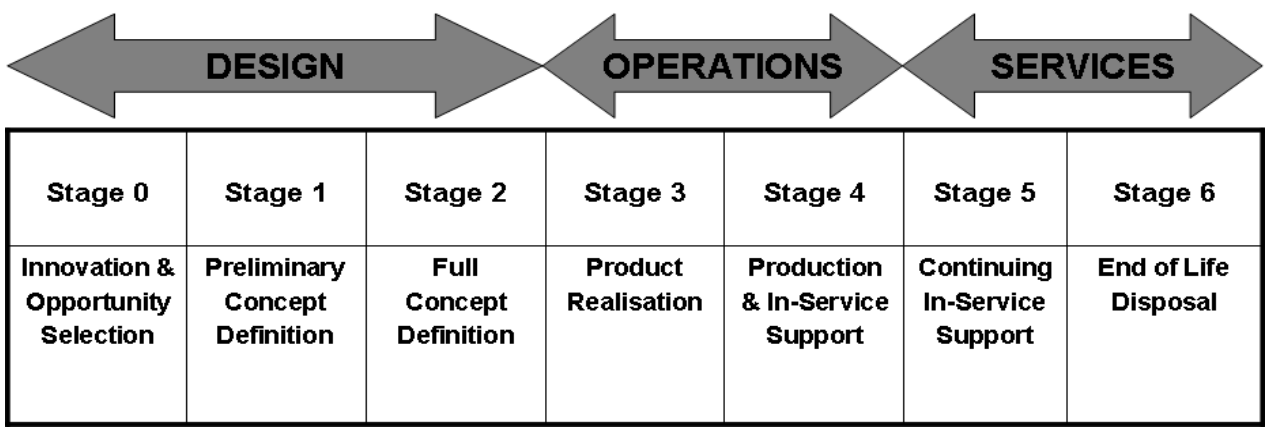

Figure 1: Rolls-Royce product development process (Rolls-Royce Plc, 2005)

To complete a stage and continue along the PDP, a series of reviews are undertaken, that include (Rolls-Royce Plc, 2005): manufacturing capability and capacity, supply chain capability and capacity, design and cost. Once stage 1 is complete a decision can be made whether to make a formal offer to the customer and continue the PDP to stage 2. 


\section{Jinks, Scanlan, Reed and Wiseall}

The product development process tries to minimise risk by reviewing and completing formal gates that must be passed before the next stage can be commenced. Reviewing a product before it is fully defined can be difficult. For instance, Figure 2 shows how the cost of a product is not fully determined until production has begun, and the majority of unit cost is unknown until the product design is frozen. Cost estimation tries to move the cost determination line towards the start of the design process, by aiding designers to analyse and understand the consequences of design decisions in terms of cost at the preliminary design stage (Sandberg Boart and Larsson, 2005).

Rush et al (2000), Curran et al (2004), Niazi et al (2006) and Tammineni (2007) have extensively reviewed cost estimation research and methods. The main cost estimating methods include: analogy, parametric, activity and feature based methods.

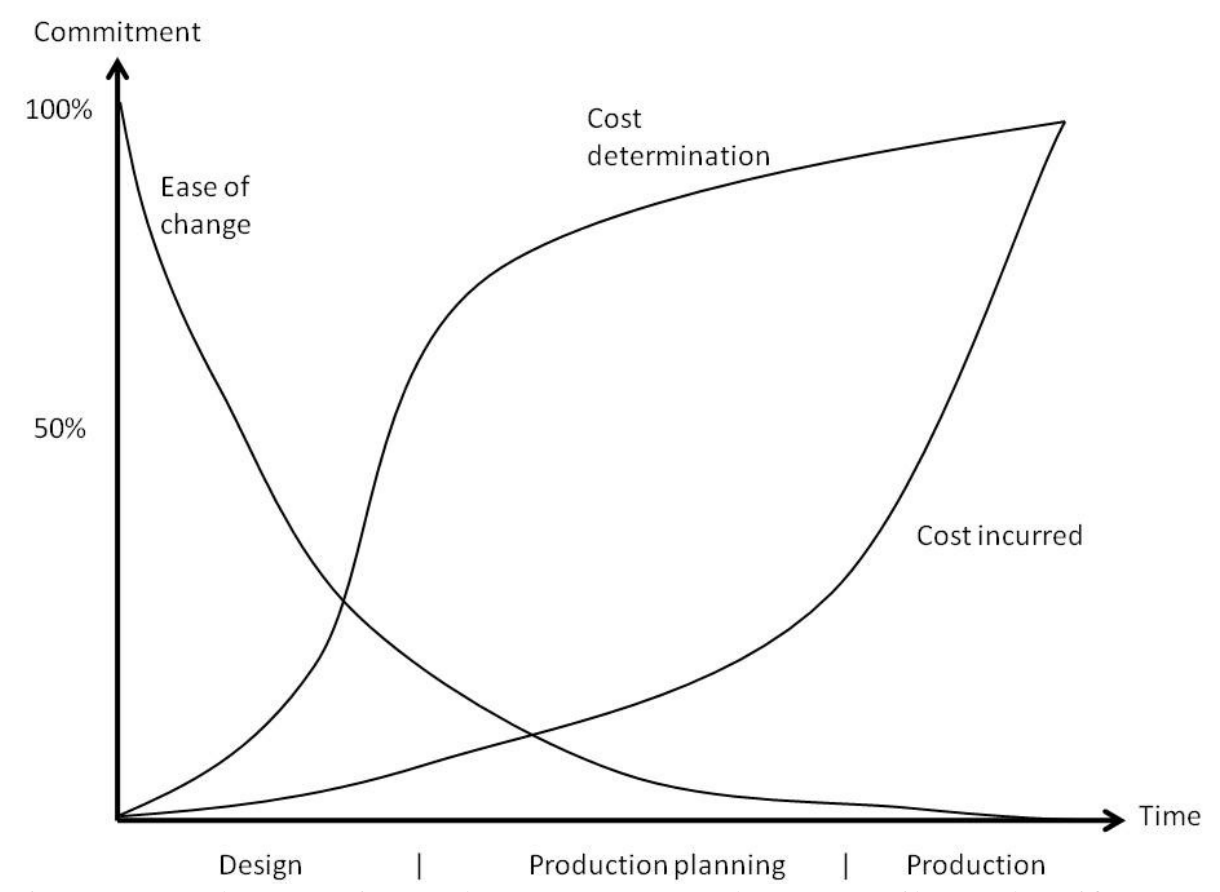

Figure 2: Product creation and costs (Layer et al., 2002, Miles and Swift, 1998)

These main cost estimation methods have various shortcomings, such as: basic visualisation in the modelling environment, little support to a user with limited programming skills, minimal ways to present manufacturing knowledge to the user and uncertainties are applied in a black box approach. These shortcomings have been addressed with the development of the knowledge based cost modelling system (Scanlan et al., 2006, Tammineni, 2007).

The knowledge based cost modelling system has addressed many shortcomings of the main cost estimating methods, but still contains limitations. The main limitation is an inability of static models to fully represent dynamic systems. A static model is defined as a representation of a system at a particular point in time (Law and Kelton, 2000). When a static model tries to represent a dynamic system, such as a factory that machines components, each operation is represented individually, and a component flows through each operation sequentially without the need to wait in a queue. A dynamic model can represent multiple components within a factory, which wait in queues, for uncertain periods of time, until machine capacity becomes available. Therefore, the complete manufacturing time is accounted for within a dynamic model.

Discrete Event Simulation (DES) is a widely used method for studying the design and operation of manufacturing systems (Creighton and Nahavandi, 2003, Knoll and Heim, 2000, Venkateswaran YoungJun and Jones, 2004). By combining DES to the knowledge based cost modelling system the next level of 
cost estimation refinement can be achieved. In order to achieve this Computer Aided Process Planning (CAPP) can be utilised.

CAPP is defined as a system that can interpret a component design in terms of features, to automate process planning of component manufacture. Process planning is defined as the preparation of a set of instructions, that detail which manufacturing processes and machines should be used, in a specific sequence, to manufacture a component design specification from raw material to finished product (Marri Gunasekaran and Grieve, 1998, Kumar and Rajotia, 2005). CAPP systems are typically used to optimise the time to manufacture a component once a component has been fully defined (Kang Han and Moon, 2003, Marri Gunasekaran and Grieve, 1998, Zhou et al., 2007).

There are two main limitations with current CAPP systems; a lack of automation (Sadaiah et al., 2002, Kang Han and Moon, 2003, Lee Jhee and Park, 2007) and unidirectional data flow (Zhou et al., 2007) from a Computer Aided Design (CAD) tool to a dedicated process planning system. If a system is to aid understanding of cost and required manufacturing resources, data should flow back to the source; the CAD tool. A bidirectional data flow should allow a design team utilising a CAD tool to understand the effect of design decisions in terms of cost and required manufacturing resources. For instance a single value or range of unit cost would allow a design team to make a decision, in terms of cost, about a component design. But, the ability to interrogate this unit cost and determine the cost drivers, would allow the design team to understand the cost and further improve it.

This paper proposes a framework and a system called DRES (Dynamic Resource Estimation System) to use dynamic DES of manufacturing processes by utilising CAD geometry and manufacturing data. The system will feedback resource based data to improve cost estimation and aid design team understanding.

The remainder of this paper is structured as follows: details of the framework and what is required in each area is described; DRES, the system being developed to implement the framework is described, with a proposed case study; and finishing with a summary and conclusions.

\section{FRAMEWORK}

To improve the current method of cost estimation for preliminary design, a new framework has been developed. The framework implements methods that aim to solve two limitations identified earlier:

- Providing a method to incorporate manufacturing simulation to improve unit cost estimation.

- Bidirectional data flow. This will aid designers to understand the consequences of design decisions in terms of cost.

The framework can be considered as five sections: geometry, component information, production process determination, factory simulation and result publishing. These sections are linked together with a knowledge repository as shown in Figure 3.

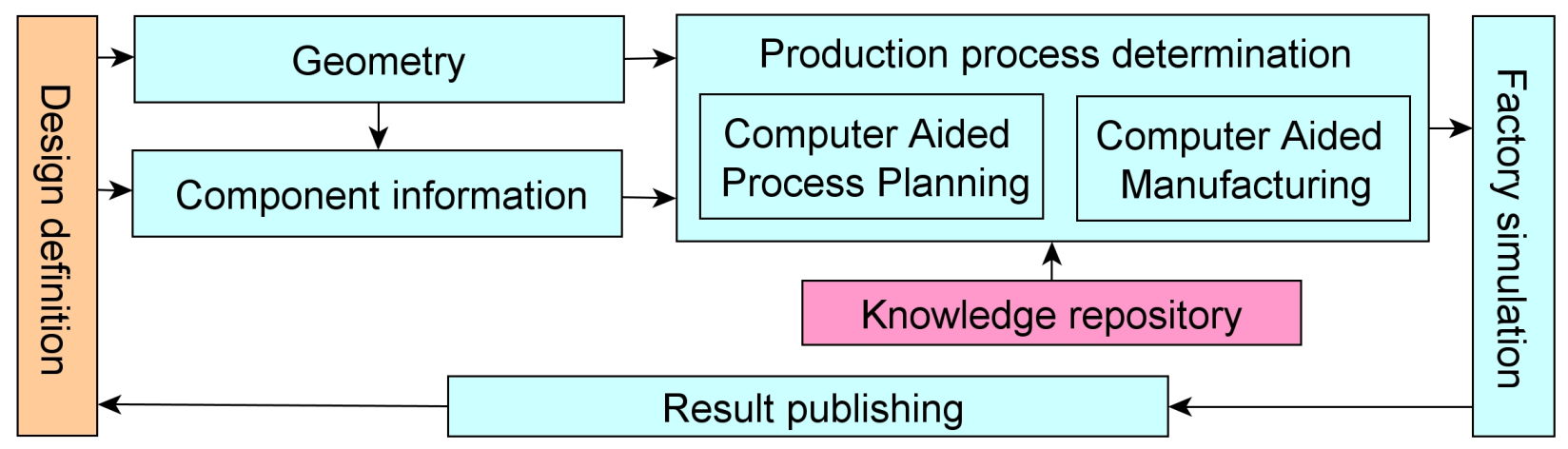

Figure 3: Framework sections

The geometry section, involves the user modifying the component geometry through the use of a CAD tool. This requires that a parameterised geometry is first created, which the user can modify within 


\section{Jinks, Scanlan, Reed and Wiseall}

the bounds of the parameterisation. When the user has completed making the necessary changes to the component geometry the user initiates DRES which implements the component information routine.

Component information, collects information about the component from the user for both the production process determination and factory simulation sections. The information collected is related to manufacturing, such as production rate, tool life requirements, and other specific questions relating to manufacturing methods.

Production process determination, uses a predefined process plan to generate manufacturing times for each viable manufacturing method. Determining a viable manufacturing method depends on three sources: component geometry, component information supplied by the user and knowledge held within a repository. A factory simulation will only take place for viable manufacturing methods. The criteria for viable manufacturing methods will depend on high level attributes, such as material compatibility, shape, size and historical precedence. When the production process has been created manufacturing operation times are calculated using all available data.

Factory simulation utilises data supplied by the production process determination section. A generic factory that corresponds to the production process is used for each manufacturing method. A generic factory model is made up of processes that are always present within a specific manufacturing method. Blocks represent the individual generic processes or machines, and data is supplied to each of these, in the form of distributed operation times from the knowledge repository. Machines have a set maximum utilisation; when a machine approaches this maximum utilisation another machine is brought online. The goal of the factory simulation is to determine the resources (number of machines and operators) required to manufacture the component at the required production rate. The results - number of machines and other resources, utilisations of each resource and times components spent in process/idle - are used by the results publishing section.

The results publishing section acts as feedback to the user. It returns resources required for the data specified by the user for each possible manufacturing method considered. If required, the user can specify a set of assumptions that allows a cost to be estimated. The user can then interrogate the cost to determine the cost drivers which could be used to optimise the component geometry.

DRES is being developed by utilising a case study to understand the data requirements of the framework. The case study will prove whether the framework and system configuration works in the correct manner, for multiple geometric values, materials and multiple manufacturing processes.

\section{DESIGN RESOURCE ESTIMATION SYSTEM (DRES)}

The preliminary system incorporates five tools: a CAD tool (Unigraphics NX (UGS NX)), a GUI written in C\#, a database (Microsoft Access), a simulation tool (Anylogic) and a static cost modelling tool (Vanguard studio). Figure 4 shows which tools are used by each framework section.

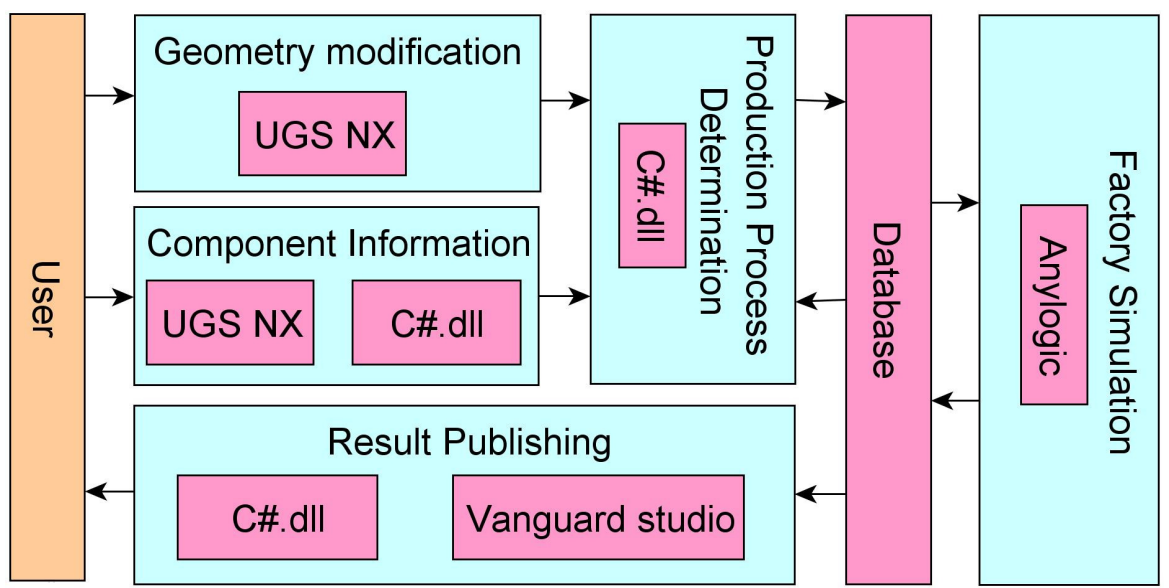

Figure 4: DRES sections and associated tools 


\section{Jinks, Scanlan, Reed and Wiseall}

UGS NX acts as the user interface and as the main tool in the tool hierarchy, as shown in Figure 5. The GUI written in C\# runs from within UGS NX. The other tools - MS Access, Anylogic and Vanguard Studio - are all initiated and run in batch mode by the C\# code.

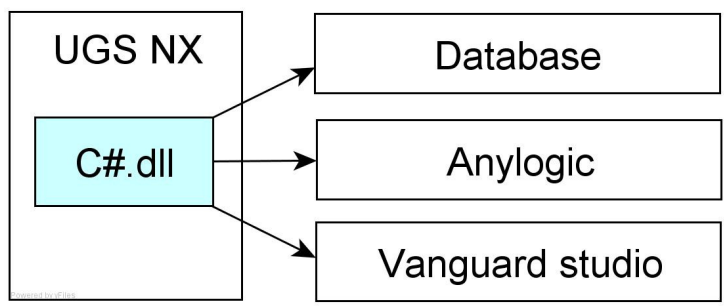

Figure 5: Tool Hierarchy

\subsection{Proposed case study}

The proposed case study is a cylindrical component that has three dimensions, inner and outer diameter and length (Figure 6). A cylinder was chosen because it is a representation of an aero engine case, which will be used for a full case study.

The case study will be able to compare manufacture of the component from three different starting shapes produced from different manufacturing methods, these include: machining from standard sized bar or tube, machining from a Ring Rolled Forging and machining from net shaped powder Hot Iso-static Pressing (HIP).

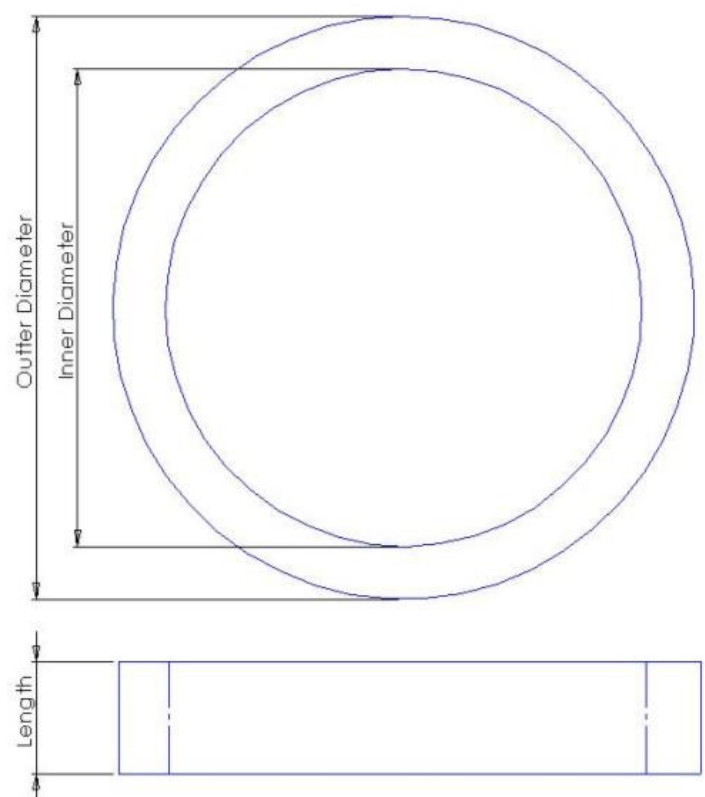

Figure 6: Case study component dimensions

Each of these starting manufacturing methods are governed by rules. For standard sized bar the diameters and length are constrained to common sizes, which, as the diameter of the component increases the availability of standard bar diameters reduces. Powder HIPing geometry is constrained by the geometry of the required pressure vessel. Ringed Rolled Forging has geometric limitations that are larger in diameter than powder HIPing but is limited in its net shape accuracy.

The geometry of the component is tested against the constraints of each manufacturing methods. If the geometry fits within the constraints a shape is created using the governing rules from each manufacturing method. In terms of the standard bar process, a diameter will be selected, from the knowledge data- 


\section{Jinks, Scanlan, Reed and Wiseall}

base, that is either $10 \mathrm{~mm}$ or $5 \%$ larger than the component diameter, depending on which is larger. The manufacturing constraints may result in a limited number of possible manufacturing methods. For instance, if the component external diameter is larger than $1.5 \mathrm{~m}$ the only possible manufacturing route, in terms of DRES, is the Ring Rolled Forging process.

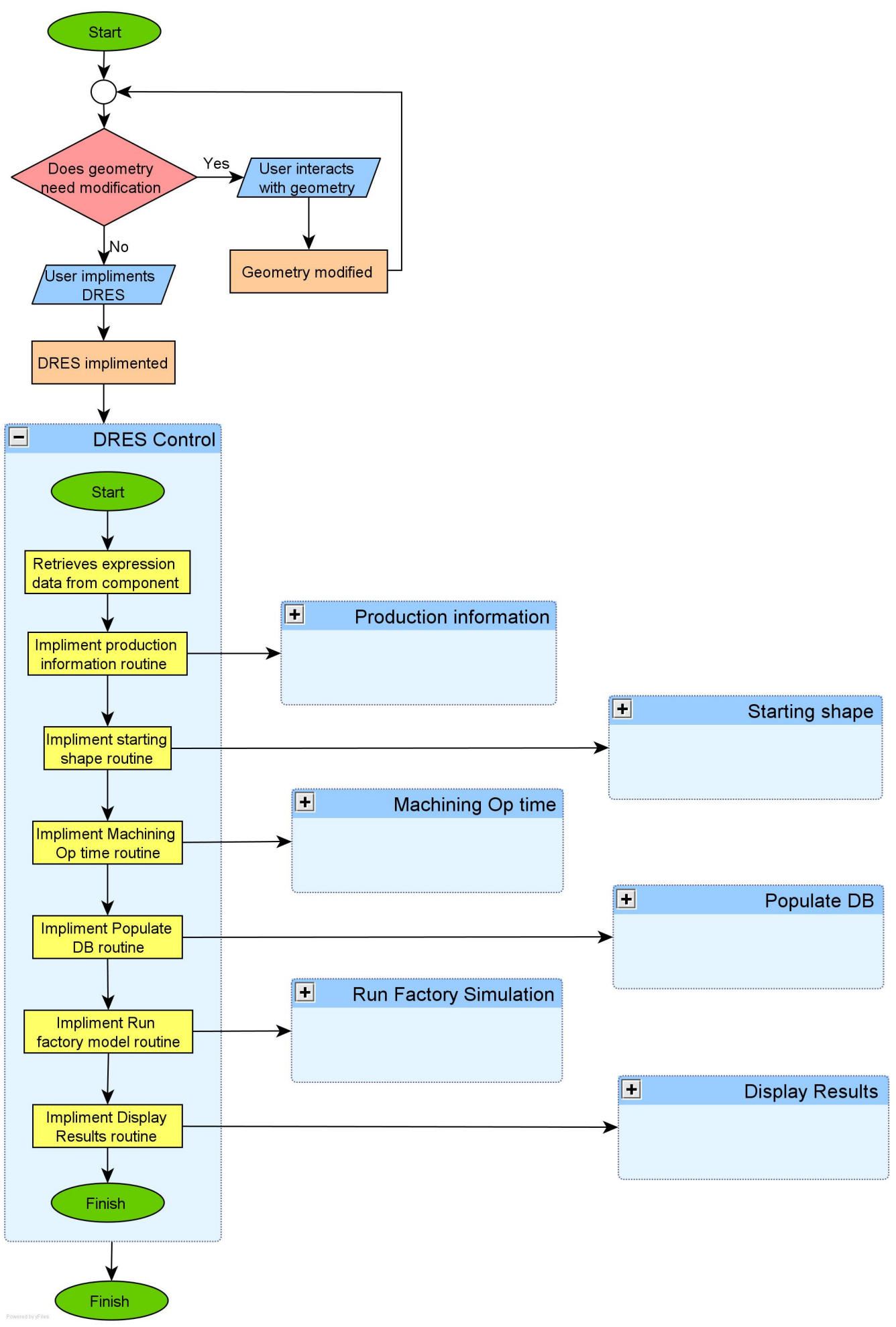

Figure 7: DRES logic 


\section{Jinks, Scanlan, Reed and Wiseall}

Figure 7 shows the high level logic that DRES follows. It can be seen that the second routine implemented is 'Starting shape'. This routine is where the shape produced by each manufacturing process, if applicable, is created. These shapes are passed onto the next routine 'Machining Op time', which determines the time to complete each machining operation, based on the geometry, and manufacturing data, such as speeds and feeds.

\section{FUTURE WORK}

A number of developments and limitations have been identified from DRES. Currently DRES has its own ability to estimate manufacturing times, but further development would seek to use a Computer Aided Manufacturing (CAM) tool, to generate the machining times. This development will be necessary to enable complex components, with multiple machining operations, to be dealt with by the system.

Manufacturing process planning is currently semi-automatically completed within DRES. For complicated components with multiple machining options, this ability, either automatic or semi-automatic, would require development in either or both standard features and Automated Feature Recognition technology (Babic Nesic and Miljkovic, 2008, Han Pratt and Regli, 2000). Therefore, for the foreseeable future a semi-defined process plan will be required for complex components.

Optimisation can be applied to factory simulation within DRES. For each design alternative, optimisation of capacity within the factory can be conducted. These optimisations would aid identification of the most cost effective manufacturing solution. Also, DRES could allow optimisation of component design. By specifying a range of possible geometric values a minimum unit cost could be found. This would allow interactions between geometry inputs to be analysed in terms of cost.

The primary objective of DRES is to provide dynamic supply chain and manufacturing simulation data to be used in unit cost estimation. DRES can be used to optimise the required resources for a specified production rate, but this represents an ideal single use factory. A more realistic utilisation of DRES would be to determine the necessary extra resources to meet a specified production rate, within a factory that is producing multiple components.

A proposed component for a full case study of DRES will be of a large civil engine casing. This case study will focus on the design decision between the traditional manufacturing route of Ring Rolled Forging, and near net shape powder HIP. This design decision is complex because of several factors:

- $\quad$ Powder HIP lead times are significantly shorter than Ring Rolled Forging lead times

- Powder HIP allows for near net shape condition of supply, thus reducing material waste and machining time

- $\quad$ Powder HIP could allow for net shape surfaces to be used

- Powder HIP requires a different manufacturing process that involves a number of batching process

- Powder HIP allows for a change in material to a higher temperature alloy. This would allow a change in design to reduce weight or increase performance

\section{SUMMARY AND CONCLUSIONS}

A review of cost estimation methods shows that many of the existing methods are based on static models. Static models can be limited in their ability to properly represent dynamic systems. It is therefore suggested that DES should be incorporated into a costing system.

To aid the incorporation of factory simulation into a costing system a direct approach has been taken. A direct coupling to a CAD tool, allows bidirectional data flow because the system is run within the CAD tool. This allows data to feedback to the design environment, the CAD tool.

This paper presents a framework specification that combines CAD, CAPP and CAM technologies with a knowledge database, to provide data for conducting manufacturing simulations and improve cost estimation. By combining these technologies with a knowledge database and a GUI, feedback of unit cost estimation and manufacturing resources can be given directly to a design team within their design envi- 


\section{Jinks, Scanlan, Reed and Wiseall}

ronment. This feedback should aid a design team to understand the consequences of design decisions in terms of cost and manufacturing resources.

A system is being developed to implement the framework and a case study of a cylindrical component will be used to prove the system. A cylinder is being used because it is representative of a proposed full case study; a large civil aero engine case.

\section{REFERENCES}

Babic, B., N. Nesic and Z. Miljkovic. 2008. A review of automated feature recognition with rule-based pattern recognition. Computers in Industry 59. 321-337.

Creighton, D. and S. Nahavandi. 2003. Application of discrete event simulation for robust system design of a melt facility. Journal of Robotics and computer integrated manufacturing 19. 469 - 477.

Curran, R., S. Raghunathan and M. Price. 2004. Review of aerospace engineering cost modelling: The genetic causal approach. Progress in Aerospace Sciences 40. 487-534.

Han, J., M. Pratt and W. C. Regli. 2000. Manufacturing feature recognition from solid models: a status report. Robotics and Automation, IEEE Transactions on 16. 782-796.

Kang, M., J. Han and J. G. Moon. 2003. An approach for interlinking design and process planning. Journal of Materials Processing Technology 139. 589-595.

Knoll, J. M. and J. A. Heim 2000 Ensuring the successful adoption of discrete event simulation in a manufacturing environment.

Kumar, M. and S. Rajotia. 2005. Development of a generative CAPP system for axisymmetric components for a job shop environment. The International Journal of Advanced Manufacturing Technology 27. 136-144.

Law, A. M. and W. D. Kelton 2000 Simulation Modeling and Analysis, $3^{\text {rd }}$ ed. Singapore: Mc Graw Hill.

Layer, A., E. T. Brinke, F. Van Houten, H. Kals and S. Haasis. 2002. Recent and future trends in cost estimation. International Journal of Computer Integrated Manufacturing 15. 499-510.

Lee, H. C., W. C. Jhee and H.-S. Park. 2007. Generative CAPP through projective feature recognition. Computers \& Industrial Engineering 53. 241-246.

Marri, H. B., A. Gunasekaran and R. J. Grieve. 1998. Computer-aided process planning: A state of art. The International Journal of Advanced Manufacturing Technology 14. 261-268.

Miles, B. L. and K. Swift. 1998. Design for Manufacture and Assembly. Manufacturing Engineering 221224.

Niazi, A., J. S. Dia, S. Balabani and L. Seneviratne. 2006. Product cost estimation: Technique classification and methodology review. Journal of Manufacturing Science and Engineering 128. 563-575.

Rolls-Royce Plc 2005 The Jet Engine, ed. London:

Rush, C. and R. Roy 2000 Analysis of cost estimation processes used within a concurrent engineering environment throughout a product life cycle. Technomic.

Sadaiah, M., D. R. Yadav, P. V. Mohanram and P. Radhakrishnan. 2002. A Generative Computer-Aided Process Planning System for Prismatic Components. The International Journal of Advanced Manufacturing Technology 20. 709-719.

Sandberg, M., P. Boart and T. Larsson. 2005. Functional Product Life-cycle Simulation Model for Cost Estimation in Conceptual Design of Jet Engine Components. Journal of Concurrent engineering: research and application 13. 331-342.

Scanlan, J., A. Rao, C. Bru, P. Hale and R. Marsh. 2006. DATUM project: cost estimating environment for support of aerospace design decision making. Journal of Aircraft 43. 1022-8.

Tammineni, S. V. 2007 Designer driven cost modelling Ph.D. Thesis, School of Engineering Sciences, University of Southampton.

Venkateswaran, J., S. Young-Jun and A. Jones 2004 Hierarchical production planning using a hybrid system dynamic-discrete event simulation architecture.

Zhou, X., Y. Qiu, G. Hua, H. Wang and X. Ruan. 2007. A feasible approach to the integration of CAD and CAPP. Computer-Aided Design 39. 324-338. 


\section{AUTHOR BIOGRAPHIES}

STUART JINKS is a EngD student in the Computational Engineering Design Research Group in the School of Engineering Sciences, University of Southampton. His research is based around unit cost estimation at preliminary design stages, specifically for novel manufacturing processes such as powder Hot Iso-static Pressing (HIP) and factory simulation. His email address is $\langle$ sj1103@soton.ac.uk $\rangle$.

JIM SCANLAN is a Professor in the Computational Engineering Design Research Group in the School of Engineering Sciences, University of Southampton. He manages a number of research programmes sponsored by BAE systems, Airbus, Rolls-Royce and the EPSRC. He has a particular interest in Design, Logistics, Simulation and Optimisation of organisations. He runs a number of courses in these disciplines as part of the Aerospace IGDS MSc programme. Jim has recently created a successful spin-off business aimed at exploiting research into design process modelling. His email address is < J.P.Scanlanesoton.ac.uk>.

PHILIPPA REED is Professor of Structural Materials, Head of the Materials Research Group and Director of the Graduate School in the School of Engineering Sciences, University of Southampton. For the past 20 years her research interests have centred on investigating micromechanisms of failure in engine and turbine materials (automotive, aerospace and power generation applications) including assessment and modelling of anomalous crack growth behaviour in a range of materials systems/architectures and the effects of external service conditions on failure processes; application of adaptive numerical modelling approaches to failure and manufacturing problems; data mining and materials data conservation; and life cycle cost modelling. Her email address is: <pasrlesoton. ac.uk>.

STEVE WISEALL is a Team Leader in the Design System Engineering - Cost Methods group, RollsRoyce plc, one of four corporate methods groups aimed at developing generic capabilities for use across the business. He has 25 years experience in Rolls-Royce mainly centred around either doing or leading capability acquisition programmes and technology transfer. He has research interests in Optimisation, Robust Design, Virtual Manufacturing, Design For Manufacture, Manufacturing Process Capability and more recently Cost Modelling and its integration into the Design process. His email address is: <steve.wiseallerolls-royce.com>. 